\title{
Towards a Generalized Stochastic Model of End-to-End Packet-Pair Sampling
}

\author{
Xiliang Liu, Member, IEEE, Kaliappa Ravindran, and Dmitri Loguinov, Member, IEEE
}

\begin{abstract}
End-to-end packet-pair probing has been used as one of the primary mechanisms to measure bottleneck capacity, crosstraffic intensity, and available bandwidth of end-to-end Internet paths. However, there has been lacking an understanding of what types of information about the path are sampled by packet-pairs and how they are encoded in the corresponding output dispersions. In this paper, we answer these questions by deriving the expression of individual output spacings (i.e., dispersions) in the context of multihop paths and general bursty cross-traffic arrivals. Based on these results, we examine stochastic properties of the sampled signals and derive the statistical mean of the output interpacket spacings as a recursive function of the input spacing. We show that the result is different from what has been obtained in prior work using fluid cross-traffic models and that this discrepancy has a significant adverse impact on the accuracy of packet-pair bandwidth measurement techniques.
\end{abstract}

Index Terms-Active measurement, bandwidth estimation, packet-pair sampling.

\section{INTRODUCTION}

$\mathbf{S}$ ENDING packet-pairs to measure network path characteristics has been a common practice in the Internet since the late 1980s. Traditionally, packet-pair dispersions are used to infer the minimum capacity of an end-to-end network path [2]-[4], [6], [9], [11], [20], [21]. More recently, people also use packetpairs to measure the cross-traffic [7], [18], [22], the raw capacity, and the residual capacity [10], [18], [23] of the available bandwidth bottleneck (i.e., the tight link).

To understand and justify packet-pair measurements, especially the recently proposed techniques that measure the tight link bandwidth characteristics, there has been a fair amount of research effort to characterize the information contained in the output dispersions of probing packet-pairs. However, previous analysis either relied on constant-rate fluid cross-traffic models [4], [17], or provided answers only partially suitable for generic bursty cross-traffic [2], [8], [19], [22]. In this paper, we provide a more accurate, yet concise characterization of packet-pair probing in the contexts of a multihop path and nonfluid cross-traffic. We identify three stochastic processes related to the cross-traffic arrival at each network hop and show that packet-pair probing essentially inspects the sample-paths

Manuscript received October 1, 2005; revised May 4, 2006.

X. Liu and K. Ravindran are with the Department of Computer Science, City University of New York, New York, NY 10016 USA (e-mail: xliu@gc.cuny.edu; ravi@cs.ccny.cuny.edu).

D. Loguinov is with the Department of Computer Science, Texas A\&M University, College Station, TX 77843 USA (e-mail: dmitri@cs.tamu.edu).

Digital Object Identifier 10.1109/JSAC.2006.884024 of these three processes and constructs the output dispersion signal based on their random sampling. We derive several closed-form expressions to describe this construction procedure and call our characterization of packet-pair probing the "sampling and constructing" model.

Under the assumption of cross-traffic stationarity, we examine the statistical properties of the signals encoded in interpacket spacings and derive the statistical mean of the output dispersions as a recursive function of the input dispersion. We show that the result substantially deviates from what was previously obtained using constant-rate fluid cross-traffic and that this deviation has a significant adverse impact on packet-pair bandwidth estimation techniques.

For the convenience of the reader, we list the terminology used in this paper in Table I. The rest of this paper is organized as follows. We summarize related work of packet-pair analysis in Section II. A detailed discussion about our "sampling-and-constructing" model to characterize packet-pair probing is given in Section III. Based on this model, we examine the statistical characteristics of the sampled signals in packet-pair dispersions in Section IV. We derive the statistical mean of output dispersions in Section V and show that its deviation from the fluid result has an adverse impact on packet-pair bandwidth estimation in Section VI. Finally, we present our concluding remarks in Section VII.

An earlier version of this work appeared in [15], where the discussion is restricted to a single-hop path.

\section{RELATED WORK}

The earliest packet-pair analysis dated back to 1988, when Jacobson [9] examined the packet-pair spacing in the absence of cross-traffic and obtained the following result:

$$
\delta^{\prime}= \begin{cases}\frac{s}{C}, & \delta \leq \frac{s}{C} \\ \delta, & \delta>\frac{s}{C}\end{cases}
$$

where $\delta$ and $\delta^{\prime}$ are the input and output spacings of the packetpair, respectively, $s$ is the probing packet size, and $C$ is the bottleneck capacity of the path. Note that when the input spacing $\delta$ is small, the output spacing $\delta^{\prime}$ contains information about $C$.

In real networks, cross-traffic is often non-negligible. To take into account the effect of cross-traffic, Dovrolis et al. [4] and Melander et al. [17] studied the relationship between the packetpair input and output rate using a constant-rate fluid cross-traffic model. In a single-hop path, their results can be summarized as follows:

$$
r^{\prime}= \begin{cases}C \frac{r}{r+\lambda}, & r \geq C-\lambda \\ r, & r<C-\lambda\end{cases}
$$


TABLE I

TERMINOLOGY

\begin{tabular}{ll}
\hline Term & Definition \\
\hline Network path & A sequence of interconnected FIFO store and forward hops \\
Hop capacity & Transmission speed of the hop in bits per second \\
Cross-traffic intensity & The average arrival rate of cross-traffic in some time interval \\
Hop available bandwidth & The hop's residual capacity after transmitting cross-traffic in some time interval \\
Path available bandwidth & The minimum hop available bandwidth along the path \\
Tight hop & The hop with the minimum available bandwidth along a path \\
Packet-pair sampled signals & The information contained in packet-pair output dispersion after passing though a link \\
\hline
\end{tabular}

where $r=s / \delta$ and $r^{\prime}=s / \delta^{\prime}$ are the input and output rates of packet-pairs, respectively, $\lambda$ is the cross-traffic intensity (i.e., arrival rate), and $C$ is again the hop capacity. Translating (2) into its spacing version, we get

$$
\delta^{\prime}= \begin{cases}\frac{\lambda}{C} \delta+\frac{s}{C}, & \delta \leq \frac{s}{C-\lambda} \\ \delta, & \delta>\frac{s}{C-\lambda}\end{cases}
$$

This shows that when the input rate is higher than hop available bandwidth, there will be a deterministic multiplicative signal of link utilization $\lambda / C$ and a deterministic additive signal $s / C$ that are sampled and encoded in the output spacing $\delta^{\prime}$.

This result can be recursively extended to a multihop path. ${ }^{1}$ Furthermore, it is easy to show using mathematical induction that the single-hop models (associated with the tight link) (2) and (3) still hold in a multihop setting when the input rate $r$ falls into a certain range

$$
r^{\prime}= \begin{cases}C_{b} \frac{r}{r+\lambda_{b}}, & C_{b}-\lambda_{b} \leq r \leq \Lambda \\ r, & r \leq C_{b}-\lambda_{b}\end{cases}
$$

where $b$ indexes the tight link, and $(0, \Lambda]$ is the input rate range where a packet-pair passing through the multihop path carries exactly the same information as it does when passing though only the tight link $b$. The exact value of $\Lambda$ depends on several factors of the path, and it is always no less than the second minimum hop available bandwidth along the path. This result leads to the recent measurement proposal TOPP [16], [18], which is a technique to infer available bandwidth and tight-link capacity.

Realistic cross-traffic is always bursty and its intensity is never a time-invariant constant. Therefore, a natural question becomes how to generalize results (1)-(4) to accommodate bursty cross-traffic. We summarize the main results of previous studies next.

To interpret his Internet measurement observations of the probing packet round-trip time (RTT) phase plot, Bolot (1993) [2] adopted a single-hop path with bursty cross-traffic in his analysis. Bolot showed that the packet-pair dispersion reflects the amount of traffic workload arrived at the router between the pair when the router does not idle between their arrivals.

$\mathrm{Hu}$ et al. (2003) [8] did a similar analysis and proposed a spacing formula under the condition when the packet-pair share the same queueing period (the same condition used in Bolot's analysis)

$$
\delta^{\prime}=\frac{s}{C}+\frac{y \delta}{C}
$$

${ }^{1}$ However, note that a recursive extension of (3) implicitly implies one-hop persistent cross-traffic routing, meaning that every cross-traffic flow only travels one hop along the path and exits the path at the next hop.

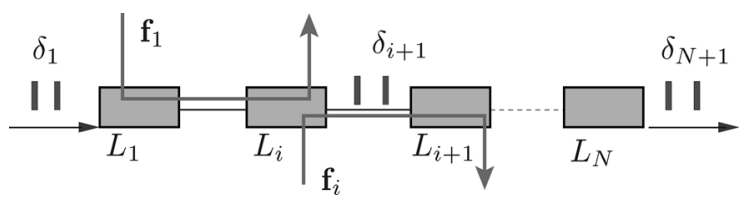

Fig. 1. A multihop model of packet pair probing.

where $y$ is a random variable reflecting the cross-traffic arrival rate in the duration between the arrivals of the probing pairs.

Both Bolot and $\mathrm{Hu}$ pointed out that when the input spacing $\delta$ is large enough, the output spacing $\delta^{\prime}$, although random, becomes equal to $\delta$ on average. In other words, the input spacing is only contaminated by some additive zero-mean signal. Kang et al. (2004) [10] further showed that the mean of the random available $y$ in (5) is equal to the long-term arrival rate of cross-traffic regardless of the stochastic nature of its arrival. We point that (5) is an important result, since almost all recently proposed packet-pair measurement techniques are based on this model.

To sum up, previous work provided an understanding of the packet-pair sampling nature when cross-traffic is constant-rate fluid. For bursty cross-traffic, existing results are both conditional (packet-pair sharing the same queueing period) and restricted into single-hop cases, and show a consistence with the fluid model where certain random terms are substituted by their means. Neither the whole picture of packet-pair sampling behavior in a practical multihop setting nor the validity of existing fluid models is clearly understood at the current stage.

\section{The "SAMPling AND CONSTRUCTING" NATURE OF PACKET-PAIR PROBING}

In this paper, we examine the nature of multihop packet-pair probing, as illustrated in Fig. 1. A packet-pair with input dispersion signal $\delta_{1}$ (or $\delta$ ) passes through an $N$-hop path. The output dispersion of the pair at link $i$ is denoted by $\delta_{i+1}$, which then serves as the input signal at link $L_{i+1}$. The pair eventually exits the path with an output dispersion $\delta_{N+1}$ (or $\delta^{\prime}$ ). Our goal in this section is to determine the path information carried by the output signal $\delta_{N+1}$. To simplify discussions, we only consider the case in which cross-traffic flows are routed along the path in a one-hop persistent fashion, meaning that flow $\mathbf{f}_{i}$ that enters the path from link $i$ will exit the path from link $i+1$. This routing pattern is amenable for recursive multihop analysis, and has been considered by several previous studies [5], [18]. For each network hop, we further assume infinite buffer capacity, first-in-first-out (FIFO) queueing, and a work-conserving discipline. For the composite of cross-traffic and probing traffic, 
we assume simple traffic arrival, i.e., at most one packet arrives at any time instant. For cross-traffic alone, we identify three sample-paths which play crucial roles in determining the nature of packet-pair probing. They are the sample-paths of cross-traffic intensity process, hop workload-difference process, and available bandwidth process. We next present a rigorous formulation for these three elements and show the basic relationship among them.

\section{A. Formulation of Cross-Traffic Arrival}

Without loss of generality, we define the relevant processes generated by the arrival of cross-traffic flow $\mathbf{f}_{i}$ at link $i$.

Definition 1: Cross-traffic flow $\mathbf{f}_{i}$ is driven by the packet counting process $\left\{N_{i}(t), 0 \leq t<\infty\right\}$ and the packet size process $\left\{S_{i}(k), 1 \leq k<\infty\right\}$. The cumulative traffic arrival at link $i$, denoted by $\left\{V_{i}(t), 0 \leq t<\infty\right\}$, is a random process counting the total volume of data (in bits) received by the hop up to time instant $t$

$$
V_{i}(t)=\sum_{k=1}^{N_{i}(t)} S_{i}(k)
$$

Definition 2: We define $\left\{Y_{i, \delta}(t), 0 \leq t<\infty\right\}$ as the process indicating the average cross-traffic arrival rate at link $i$ in the interval $(t, t+\delta]$

$$
Y_{i, \delta}(t)=\frac{V_{i}(t+\delta)-V_{i}(t)}{\delta}
$$

and call it " $\delta$-interval cross-traffic intensity process at link $i . "$

The second critical element is hop workload-difference process.

Definition 3: Hop workload process $\left\{W_{i}(t), 0 \leq t<\infty\right\}$ indicates the sum at time instant $t$ of service times of all packets in the queue and the remaining service time of the packet in service at link $i$.

Definition 4: We define $\left\{D_{i, \delta}(t), 0 \leq t<\infty\right\}$ as the process indicating the difference between the workload of link $i$ at time $t$ and $t+\delta$

$$
D_{i, \delta}(t)=W_{i}(t+\delta)-W_{i}(t)
$$

and call it " $\delta$-interval workload-difference process at link $i$."

The third important process is the available bandwidth process.

Definition 5: Hop utilization process $\left\{U_{i}(t), 0 \leq t<\infty\right\}$ at link $i$ is an on-off process associated with $\left\{W_{i}(t)\right\}$

$$
U_{i}(t)= \begin{cases}1, & W_{i}(t)>0 \\ 0, & W_{i}(t)=0\end{cases}
$$

and $\delta$-interval hop idle process

$$
I_{i}(t, t+\delta)=I_{i, \delta}(t)=\delta-\int_{t}^{t+\delta} U_{i}(x) d x
$$

is a process indicating the total amount of idle time of the forwarding hop in $[t, t+\delta]$. We further call time interval $[t, t+\delta]$ a "hop busy period" if $I_{i, \delta}(t)=0$ and a "hop idle period" if $I_{i, \delta}(t)=\delta$.
Definition 6: We define $\left\{B_{i, \delta}(t), 0 \leq t<\infty\right\}$ as the process indicating the residual bandwidth at link $i$ in the time interval $[t, t+\delta]$

$$
B_{i, \delta}(t)=C_{i}\left(1-\frac{1}{\delta} \int_{t}^{t+\delta} U_{i}(x) d x\right)=\frac{I_{i, \delta}(t) C_{i}}{\delta}
$$

and call it " $\delta$-interval available bandwidth process" at link $i$.

The following theorem describes the relationship among the three important processes.

Theorem 1: For all positive $\mathrm{t}$ and $\delta$, the following holds:

$$
B_{i, \delta}(t)=C_{i}-Y_{i, \delta}(t)+\frac{D_{i, \delta}(t) C_{i}}{\delta} .
$$

Proof: Note that the total hop idle time of link $i$ within the time interval $[t, t+\delta]$ is

$$
I_{i, \delta}(t)=\frac{B_{i, \delta}(t) \delta}{C}
$$

The amount of data transmitted by the hop within the time interval $[t, t+\delta]$ is

$$
\begin{aligned}
V_{i}(t+\delta)-V_{i}(t)+\left(W_{i}(t)-W_{i}(t+\delta)\right) & C_{i} \\
& =Y_{i, \delta}(t) \delta-D_{i, \delta}(t) C .
\end{aligned}
$$

Thus, the hop working time is

$$
\frac{Y_{i, \delta}(t) \delta}{C_{i}}-D_{i, \delta}(t)
$$

Since $\delta$ is the sum of hop working time and hop idle time. Adding up (10) and (11), we get

$$
\delta=\frac{B_{i, \delta}(t) \delta}{C_{i}}-D_{i, \delta}(t)+\frac{Y_{i, \delta}(t) \delta}{C_{i}} .
$$

Rearranging (12), we get the desired result.

A packet-pair arriving at link $i$ with dispersion $\delta_{i}$ interacts with the sample-paths of the $\delta_{i}$-interval processes we just formulated. We next examine certain details about this interaction.

\section{B. Probing Intrusion of Packet-Pairs}

We use the triple $\left\langle a_{i}, \delta_{i}, s\right\rangle$ to denote a pair of probing packets $p_{1}$ and $p_{2}$ of the same size that arrive at link $i$ at time instant $a_{i}$. The first element $a_{i}$ in the triple is the arrival time of the packet $p_{1}$ to the hop; $\delta_{i}$ is the interpacket spacing; and $s$ is the probing packet size. The arrival time of $p_{2}$ is then $a_{i}+\delta_{i}$. The departure time of $p_{1}$ from link $i$ is denoted by $d_{i}$ and the output spacing between $p_{1}$ and $p_{2}$ is $\delta_{i+1}$. This means that $p_{2}$ departs from link $i$ at time $d_{i}+\delta_{i+1}$. In terms of rate, the input and output probing rates are $r_{i}=s / \delta_{i}$ and $r_{i+1}=s / \delta_{i+1}$.

We use $\tilde{W}_{i}(t)$ and $\tilde{I}_{i, \delta}(t)$ to denote the workload process and the hop idle process associated with the superposition of crosstraffic and the probing packet-pair. Note that traffic composition only increases hop workload. That is, for all $t, \tilde{W}_{i}(t) \geq W_{i}(t)$. Therefore, we define the following function to help understand this intrusion behavior of packet probing. 


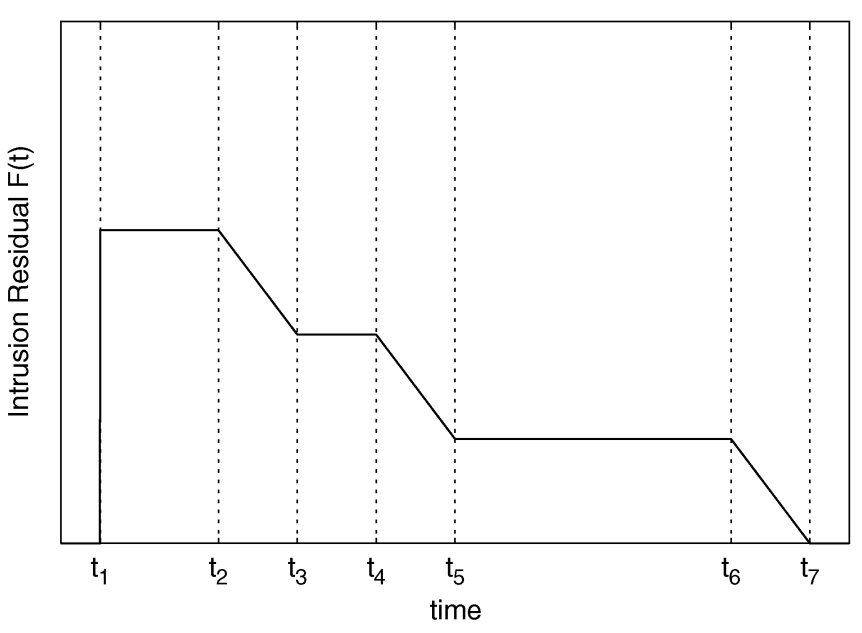

Fig. 2. Illustration of the probing intrusion behavior of a single packet.

Definition 7: The intrusion range of a probing traffic into $W_{i}(t)$ is the set $\left\{t: \tilde{W}_{i}(t)>W_{i}(t)\right\}$. The intrusion residual function is $F(t)=\tilde{W}_{i}(t)-W_{i}(t)$.

Let us next examine how the arrival of a single probing packet changes the hop workload. Note that before the arrival of the probing packet, $\tilde{W}_{i}(t)=W_{i}(t)$. The workload gets an immediate increment of $s / C_{i}$ upon the packet arrival, where $s$ is the packet size. In $W_{i}(t)$ 's busy periods, the workload difference $F(t)$ remains unchanged. In $W_{i}(t)$ 's idle periods, The workload difference $F(t)$ decreases linearly with slope -1 until it becomes 0 , which marks the end of the intrusion range. Fig. 2 illustrates this behavior, from which we can infer that $\left(t_{1}, t_{2}\right)$, $\left(t_{3}, t_{4}\right)$ and $\left(t_{5}, t_{6}\right)$ are three busy periods in $W(t)$, whereas $\left(t_{2}, t_{3}\right),\left(t_{4}, t_{5}\right)$, and $\left(t_{6}, t_{7}\right)$ are three idle periods in $W(t)$. Time instant $t_{1}$ is the arrival time of the probing packet, whereas $t_{7}$ is the end point of the intrusion range. ${ }^{2}$

When $W_{i}(t)$ is probed by a packet-pair $\left\langle a_{i}, \delta_{i}, s\right\rangle$, the first probing packet $p_{1}$ experiences a queueing delay of $W_{i}\left(a_{i}\right)$. The queueing delay of the second probing packet $p_{2}$ is however not $W_{i}\left(a_{i}+\delta_{i}\right)$, but the left-hand limit $^{3} \tilde{W}_{i}\left(a_{i}+\delta_{i}-\right)$. There is some extra amount of queueing delay, besides the portion that comes from the original hop workload $W_{i}\left(a_{i}+\delta_{i}\right)$, that $p_{2}$ will experience. We denote this extra amount of queueing delay by $R_{i, \delta_{i}}\left(a_{i}\right)$. That is

$$
R_{i, \delta_{i}}\left(a_{i}\right)=\tilde{W}_{i}\left(a_{i}+\delta_{i}-\right)-W\left(a_{i}+\delta_{i}\right)
$$

As a direct observation from the intrusion behavior illustrated in Fig. 2, the term $R_{i, \delta_{i}}\left(a_{i}\right)$ can be computed as follows:

$$
R_{i, \delta_{i}}\left(a_{i}\right)=\left(\frac{s}{C_{i}}-I_{i, \delta_{i}}\left(a_{i}\right)\right)^{+}=\left(\frac{s-B_{i, \delta_{i}}\left(a_{i}\right) \delta_{i}}{C_{i}}\right)^{+}
$$

where $X^{+}=\max (X, 0)$.

\footnotetext{
${ }^{2}$ Note that the probing packet departs before $t_{7}$.

${ }^{3}$ Note that the queueing delay of $p_{2}$ is not $\tilde{W}_{i}\left(a_{i}+\delta_{i}\right)$, since the arrival of $p_{2}$ itself causes a sudden increment of workload at time $a_{i}+\delta_{i}$. This makes a difference of $s / C_{i}$ between $\tilde{W}_{i}\left(a_{i}+\delta_{i}\right)$ and $\tilde{W}_{i}\left(a_{i}+\delta_{i}-\right)$.
}

We are also interested in computing $\tilde{I}_{i, \delta_{i}}\left(a_{i}\right)$ when the hop is probed by packet-pair $\left\langle a_{i}, \delta_{i}, s\right\rangle$, which can be expressed as

$$
\tilde{I}_{i, \delta_{i}}\left(a_{i}\right)=\left(I_{i, \delta_{i}}\left(a_{i}\right)-\frac{s}{C_{i}}\right)^{+}=\left(\frac{B_{i, \delta_{i}}\left(a_{i}\right) \delta_{i}-s}{C_{i}}\right)^{+}
$$

Notice that between the two terms $R_{i, \delta_{i}}\left(a_{i}\right)$ and $\tilde{I}_{i, \delta_{i}}\left(a_{i}\right)$, there is at most one positive term for any given $a_{i}$. When $R_{i, \delta_{i}}\left(a_{i}\right)>0$, the two packets in the pair share the same hop busy period and $\tilde{I}_{i, \delta_{i}}\left(a_{i}\right)=0$. When $\tilde{I}_{i, \delta_{i}}\left(a_{i}\right)>0$, the two packets fall into different hop busy period and $R_{i, \delta_{i}}\left(a_{i}\right)=0$.

We are now ready to derive the recursive relation between the input spacing $\delta_{i}$ and the output spacing $\delta_{i+1}$ for an individual packet-pair. This relation is a milestone of our packetpair analysis.

\section{Output Packet-Pair Dispersion}

We first present a corollary, which is due to the work-conserving assumption.

Corollary 1: For any packet arriving into the hop at time $t_{1}$ and departing from the hop at time $t_{2}$, the time interval $\left[t_{1}, t_{2}\right]$ is a hop busy period.

This corollary immediately leads to the following lemma.

Lemma 1: When link $i$ is probed by a packet-pair $\left\langle a_{i}, \delta_{i}, s\right\rangle$, we have $\tilde{I}_{i}\left(d_{i}, d_{i}+\delta_{i+1}\right)=\tilde{I}_{i}\left(a_{i}, a_{i}+\delta_{i}\right)$.

Proof: First, due to Corollary 1, we have

$$
\tilde{I}_{i}\left(a_{i}, d_{i}\right)=\tilde{I}_{i}\left(a_{i}+\delta_{i}, d_{i}+\delta_{i+1}\right)=0
$$

Further, notice that $\tilde{I}_{i}\left(a_{i}, d_{i}+\delta_{i+1}\right)$ can be expressed in the following two ways:

$$
\begin{aligned}
& \tilde{I}_{i}\left(a_{i}, d_{i}+\delta_{i+1}\right) \\
& \quad=\tilde{I}_{i}\left(a_{i}, d_{i}\right)+\tilde{I}_{i, \delta_{i+1}}\left(d_{i}\right)=\tilde{I}_{i, \delta_{i+1}}\left(d_{i}\right) \\
& \quad=\tilde{I}_{i, \delta_{i}}\left(a_{i}\right)+\tilde{I}_{i}\left(a_{i}+\delta_{i}, d_{i}+\delta_{i+1}\right)=\tilde{I}_{i, \delta_{i}}\left(a_{i}\right) .
\end{aligned}
$$

Combining (17) and (18), we have $\tilde{I}_{i}\left(d_{i}, d_{i}+\delta_{i+1}\right)=$ $\tilde{I}_{i}\left(a_{i}, a_{i}+\delta_{i}\right)$.

Our next theorem expresses the output spacing of a packetpair at link $i$ from two different angles.

Theorem 2: When link $i$ is probed by a packet pair $\left\langle a_{i}, \delta_{i}, s\right\rangle$, the output spacing $\delta_{i+1}$ can be expressed as

$$
\begin{aligned}
\delta_{i+1} & =\frac{Y_{i, \delta_{i}}\left(a_{i}\right) \delta_{i}}{C_{i}}+\frac{s}{C_{i}}+\left(\frac{B_{i, \delta_{i}}\left(a_{i}\right) \delta_{i}-s}{C_{i}}\right)^{+} \\
& =\delta_{i}+D_{i, \delta_{i}}\left(a_{i}\right)+\left(\frac{s-B_{i, \delta_{i}}\left(a_{i}\right) \delta_{i}}{C_{i}}\right)^{+} .
\end{aligned}
$$

Proof: We examine the hop activity with respect to $\tilde{W}_{i}(t)$ within the time interval $\left[d_{i}, d_{i}+\delta_{i+1}\right]$. Notice that $s / C_{i}$ time units are spent on serving probing packet $p_{2}$ and that

$$
\frac{V_{i}\left(a_{i}+\delta_{i}\right)-V\left(a_{i}\right)}{C_{i}}=\frac{Y_{i, \delta_{i}}\left(a_{i}\right) \delta_{i}}{C_{i}}
$$




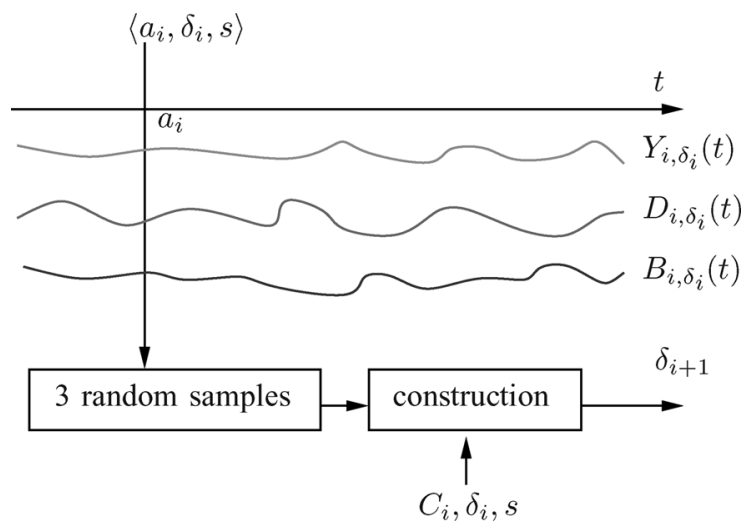

Fig. 3. The "sampling-and-constructing" nature of packet pair probing.

time units are spent on serving the cross-traffic that has arrived to the hop during the time interval $\left(a_{i}, a_{i}+\delta_{i}\right)$. Thus, the total hop working time in $\left[d_{i}, d_{i}+\delta_{i+1}\right]$ is given by

$$
\frac{Y_{i, \delta_{i}}\left(a_{i}\right) \delta_{i}}{C_{i}}+\frac{s}{C_{i}}
$$

Also, notice that $\tilde{I}\left(d_{i}, d_{i}+\delta_{i+1}\right)$ is the total idle time of the hop during this time interval. Since the sum of the hop working time in (21) and hop idle time must be equal to $\delta_{i+1}$, we immediately have the following:

$$
\delta_{i+1}=\frac{Y_{i, \delta_{i}}\left(a_{i}\right) \delta_{i}}{C_{i}}+\frac{s}{C_{i}}+\tilde{I}\left(d_{i}, d_{i}+\delta_{i+1}\right) .
$$

Further, due to Lemma 1 and (15), we get

$$
\tilde{I}\left(d_{i}, d_{i}+\delta_{i+1}\right)=\tilde{I}_{\delta_{i}}\left(a_{i}\right)=\left(\frac{B_{i, \delta_{i}}\left(a_{i}\right) \delta_{i}-s}{C_{i}}\right)^{+} .
$$

Substituting (23) back to (22), we proved the first equality in (19). For the second part of (19), first notice that the total delays of $p_{1}$ and $p_{2}$ at link $i$ are given by

$$
\begin{aligned}
d_{i}-a_{i} & =W_{i}\left(a_{i}\right)+\frac{s}{C_{i}}, \\
\left(d_{i}+\delta_{i+1}\right)-\left(a_{i}+\delta_{i}\right) & =R_{i, \delta_{i}}\left(a_{i}\right)+W_{i}\left(a_{i}+\delta_{i}\right)+\frac{s}{C_{i}} .
\end{aligned}
$$

Subtracting the delay of $p_{1}$ from that of $p_{2}$, we get

$$
\delta_{i+1}=\delta_{i}+R_{i, \delta_{i}}\left(a_{i}\right)+D_{i, \delta_{i}}\left(a_{i}\right) .
$$

Substituting (14) into (24), we get the second half of (19).

The most salient feature of Theorem 2 is that the result is unconditional, in the sense that it neither relies on any assumption on cross-traffic arrival pattern nor imposes any restriction on the input signal $\delta_{i}$. In addition, this result enforces such a conceptual idea that packet-pair probing can be viewed as a "sampling-and-constructing" procedure, as illustrated in Fig. 3. The packet-pair $\left\langle a_{i}, \delta_{i}, s\right\rangle$ is essentially sampling the three sample- paths $Y_{i, \delta_{i}}(t), D_{i, \delta_{i}}(t)$, and $B_{i, \delta_{i}}(t)$ at the time point $a_{i}$, and then constructing the output signal $\delta_{i+1}$ using the three samples based on (19). Although (19) shows two different ways of constructing the output signal, they both produce the same result. We surely can take advantage of Theorem 1 and rewrite (19) in a form involving only two processes (e.g., $Y_{i, \delta_{i}}(t)$ and $D_{i, \delta_{i}}(t)$ ). However, the present version is more intuitive and makes later analysis easier. Our characterization already sheds light on what the sampled path information is and how it is encoded in the output dispersion signal. It also allows investigation of the statistical nature of the sampled information from an analytical angle rather than experimental observations.

\section{The Statistics of Packet-PAir SAmPled Signals}

In this section, we study the statistical properties of the packet-pair sampled signals at link $i$. We classify the sampled signals into two categories. The first category includes the directly sampled signals such as $Y_{i, \delta_{i}}\left(a_{i}\right), D_{i, \delta_{i}}\left(a_{i}\right)$, and $B_{i, \delta_{i}}\left(a_{i}\right)$, which are inherent to the queueing system and are independent of the input packet-pair parameters. The second category contains the derived signals such as $R_{i, \delta_{i}}\left(a_{i}\right)$ and $\tilde{I}_{i, \delta_{i}}\left(a_{i}\right)$, whose properties are related to both the system being sampled and the input packet-pair parameters. We focus on the case when $\delta_{i}=\delta$ is a deterministic constant. This simple case is important for two reasons. First, in a single-hop path, the input dispersion is formed by the probing source and consequently is a deterministic constant. Second, even when $\delta_{i}$ is a random output from link $i-1$, the analysis of the sampled signals would rely on conditioning on $\delta_{i}$, which requires understanding the situation when $\delta_{i}$ is a constant.

\section{A. Directly Sampled Signals}

To simplify discussions, we make a stationarity assumption on cross-traffic arrival in flow $\mathbf{f}_{i}$ so that $Y_{i, \delta}(t), D_{i, \delta}(t)$, and $B_{i, \delta}(t)$ can be treated as identically distributed processes. ${ }^{4}$

Assumption 1: The cumulative traffic arrival process $\left\{V_{i}(t)\right\}$ of flow $\mathbf{f}_{i}$ has ergodic stationary increments, i.e., for any positive $\delta$, the process $\left\{Y_{i, \delta}(t)\right\}$ is an identically distributed process with ensemble mean $\lambda_{i}<C_{i}$.

This assumption imposes two restrictions on the process $\left\{Y_{i, \delta}(t)\right\}$. First, the stationarity assumption implies that $Y_{i, \delta}(t)$ has time-invariant distribution and that its marginal distribution at any time instant $t$ can be described by the same random variable $Y_{i, \delta}$. Second, the ergodicity assumption implies that the variance of the random variable $Y_{i, \delta}$ decays to 0 as $\delta$ increases.

We also assume that the queueing system at link $i$ has evolved for a sufficiently long time and has entered its equilibrium state. Therefore, the hop workload process $\left\{W_{i}(t)\right\}$ also exhibits distributional stationarity. This property is further carried over to process $\left\{D_{i, \delta}(t)\right\}$ and $\left\{B_{i, \delta}(t)\right\}$, whose time-invariant marginal distributions are characterized by their corresponding random variables $D_{i, \delta}$ and $B_{i, \delta}$, respectively.

The following lemma summarizes the first-order statistics of the three sampled signals. They are all immediate consequences of the cross-traffic stationarity stated in Assumption 1.

\footnotetext{
${ }^{4}$ We impose no constraint on their correlation structures, however.
} 


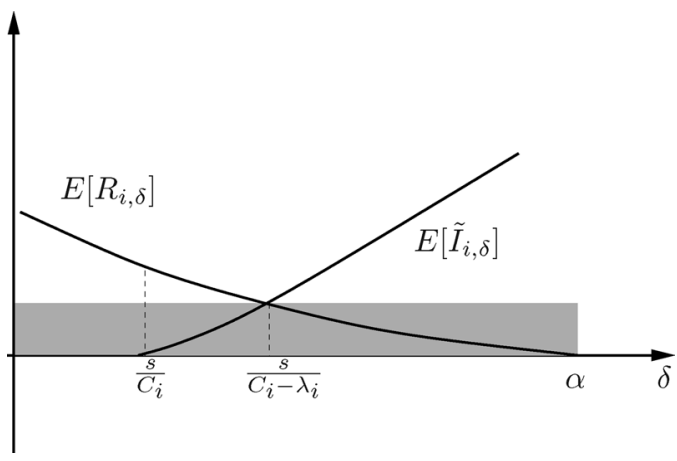

Fig. 4. The evolving trend of $E\left[\tilde{I}_{i, \delta_{i}}\right]$ and $E\left[R_{i}, \delta\right]$ with respect to $\delta$, while keeping $s$ constant.

Lemma 2: For any positive $\delta$, the ensemble means of the random variables $Y_{i, \delta}, D_{i, \delta}$, and $B_{i, \delta}$ are given by

$$
\left\{\begin{array}{l}
E\left[Y_{i, \delta}\right]=\lambda_{i} \\
E\left[D_{i, \delta}=0\right. \\
E\left[B_{i, \delta}=C_{i}-\lambda_{i}\right.
\end{array} .\right.
$$

For the second-order statistics of these signals, note that similar to $Y_{i, \delta}$, the variance of $B_{i, \delta}$ also decays to 0 (due to the cross-traffic ergodicity) when the sampling interval $\delta$ becomes large. ${ }^{5}$ This further implies that the distribution of $B_{i, \delta}$ converges to the following step function, as $\delta \rightarrow \infty$ :

$$
P_{\infty}(x)= \begin{cases}0, & x<C_{i}-\lambda_{i} \\ 1, & x \geq C_{i}-\lambda_{i}\end{cases}
$$

\section{B. Derived Signals}

We now study the first-order statistics of the two derived signals $R_{i, \delta}\left(a_{i}\right)$ and $\tilde{I}_{i, \delta}\left(a_{i}\right)$. Recall that both terms are functionally related to $B_{i, \delta}\left(a_{i}\right)$, as shown in (14) and (15). Due to the distributional stationarity of $B_{i, \delta}(t)$, the two derived processes $R_{i, \delta}(t)$ and $\tilde{I}_{i, \delta}(t)$ also have time-invariant marginal distributions, described by the random variables $R_{i, \delta}$ and $\tilde{I}_{i, \delta}$, respectively. Denoting by $P_{\delta}(x)$ the distribution function of $B_{i, \delta}$, the ensemble means of the two derived signals can be computed as follows:

$$
\begin{aligned}
E\left[R_{i, \delta}\right] & =\int_{0}^{s / \delta} \frac{s-x \delta}{C_{i}} d P_{\delta}(x) \\
E\left[\tilde{I}_{i, \delta}\right] & =\int_{s / \delta}^{C_{i}} \frac{x \delta-s}{C_{i}} d P_{\delta}(x) .
\end{aligned}
$$

Unlike the three directly sampled signals, the statistical means of the derived signals are dependent on $\delta$. Fig. 4 plots the evolving trend of the two means with respect to $\delta$, while keeping $s$ constant. As shown in the figure, $E\left[R_{i, \delta}\right]$ shows a monotonically decreasing trend as $\delta$ increases and at some

\footnotetext{
${ }^{5}$ On the other hand, notice that $D_{i, \delta}$ is not a moving average by nature. Hence, the variance of $D_{i, \delta}$ would not decay with the increase of $\delta$. Instead, it converges to $2 \operatorname{Var}\left[W_{i}\right]$, i.e., twice as much as the hop workload variance.
}

point $\alpha$ it becomes 0 or practically negligible. The other term $E\left[\tilde{I}_{i, \delta}\right]$ remains 0 for $\delta \leq s / C_{i}$ and then shows a monotonically increasing trend, asymptotically approaching a linear function of $\delta$. The two curves intersect at the point $\delta=s /\left(C_{i}-\lambda_{i}\right)$. These results can be summarized into the following two set of formulas:

$$
\begin{aligned}
& \begin{cases}E\left[\tilde{I}_{i, \delta}\right]=0, & \delta \leq \frac{s}{C_{i}} \\
E\left[\tilde{I}_{i, \delta}\right]=E\left[R_{i, \delta}\right], & \delta=\frac{s}{C_{i}-\lambda_{i}} \\
E\left[R_{i, \delta}\right]=\frac{s}{C_{i}}-\frac{C_{i}-\lambda_{i}}{C_{i}} \delta, & \delta \leq \frac{s}{C_{i}}\end{cases} \\
& \left\{\begin{array}{l}
\lim _{\delta \rightarrow \infty} E\left[\tilde{I}_{i, \delta}\right]=\frac{C_{i}-\lambda_{i}}{C_{i}} \delta-\frac{s}{C_{i}} \\
\lim _{\delta \rightarrow \infty} E\left[R_{i, \delta}\right]=0
\end{array}\right. \\
& \lim _{\delta \rightarrow s / C_{i}} E\left[\tilde{I}_{i, \delta}\right]=0
\end{aligned}
$$

All these properties are easily provable based on the statistical characteristics of $B_{i, \delta}$. We next prove one of them and leave the verification of the others to the reader.

Theorem 3: The term $E\left[R_{i, \delta}\right]$ is a continuous and monotonic decreasing function of $\delta$ in the range $(0, \infty)$. It converges to 0 as $\delta$ increases.

Proof: First, note that for any $\Delta>0$ and any $t$, we have

$$
0 \leq R_{i, \delta}(t)-R_{i, \delta+\Delta}(t) \leq \Delta .
$$

This difference defines a new random variable, whose ensemble falls into the range $[0, \Delta]$

$$
0 \leq E\left[R_{i, \delta}(t)-R_{i, \delta+\Delta}(t)\right] \leq \Delta
$$

which can be rewritten as

$$
0 \leq E\left[R_{i, \delta}\right]-E\left[R_{i, \delta+\Delta}\right] \leq \Delta
$$

where we have dropped $t$ because of the stationarity of the derived process $R_{i, \delta}(t)$. This result shows that $E\left[R_{i, \delta}\right]$ is a monotonic decreasing function of $\delta$. Further, by taking the limit of (33) when $\Delta \rightarrow 0$, we have

$$
\lim _{\Delta \rightarrow 0}\left(E\left[R_{\delta}(t)\right]-E\left[R_{\delta+\Delta}(t)\right]\right)=0 .
$$

This proves the continuity of $E\left[R_{i, \delta}\right]$ with respect to $\delta$ in the range $(0, \infty)$. Next, we show its convergence to 0 as $\delta$ increases. First, recalling (27), we have

$$
\begin{aligned}
\lim _{\delta \rightarrow \infty} E\left[R_{i, \delta}\right] \\
=\lim _{\delta \rightarrow \infty} \int_{0}^{s / \delta} \frac{s-x \delta}{C_{i}} d P_{\delta}(x) \\
=\lim _{\delta \rightarrow \infty} \int_{0}^{s / \delta} \frac{s}{C_{i}} d P_{\delta}(x)-\lim _{\delta \rightarrow \infty} \int_{0}^{s / \delta} \frac{x \delta}{C_{i}} d P_{\delta}(x) .
\end{aligned}
$$


Note that the first item in (35) is zero ${ }^{6}$

$$
\lim _{\delta \rightarrow \infty} \int_{0}^{s / \delta} \frac{s}{C_{i}} d P_{\delta}(x)=\lim _{\delta \rightarrow \infty} \frac{s}{C_{i}} P_{\delta}\left(\frac{s}{\delta}\right)=\frac{s}{C_{i}} P_{\infty}(0)=0
$$

and the second item in (35) is also zero

$$
\begin{aligned}
& 0 \leq \lim _{\delta \rightarrow \infty} \int_{0}^{s / \delta} \frac{x \delta}{C_{i}} d P_{\delta}(x) \leq \lim _{\delta \rightarrow \infty} \int_{0}^{s / \delta} \frac{s}{\frac{\delta}{C_{i}}} d P_{\delta}(x) \\
& =\lim _{\delta \rightarrow \infty} \frac{s}{C_{i}} P_{\delta}\left(\frac{s}{\delta}\right)=\frac{s}{C_{i}} P_{\infty}(0)=0 .
\end{aligned}
$$

Hence, the limit of $E\left[R_{i, \delta}\right]$ when $\delta \rightarrow \infty$ is zero.

Before we conclude this section, we briefly mention the firstorder statistics of the sampled signals when the input packet-pair dispersion $\delta_{i}$ is random. By conditioning on $\delta_{i}$, it is easy to show that the randomness of $\delta_{i}$ does not at all change the mean of all three directly sampled signals. We still have that $E\left[Y_{i, \delta_{i}}\right]=\lambda_{i}$, $E\left[D_{i, \delta_{i}}\right]=0$, and $E\left[B_{i, \delta_{i}}\right]=C_{i}-\lambda_{i}$. For the derived signals, we have to know the distribution of $\delta_{i}$ to compute their ensemble means. However, it is important to keep in mind that the two derived signals always have non-negative means, regardless of whether the input dispersion $\delta_{i}$ is random or not.

\section{Packet-Pair Probing Response Curves}

In this section, we derive the statistical mean of packet-pair output signals (dispersion or rate) as a function of the input signals. This function, which we call the packet-pair probing response curve, serves as a theoretical foundation for bandwidth measurement tools such as TOPP [18] and Spruce [23]. Previous derivations assumed constant-rate fluid cross-traffic and obtained (2) and (3). In this section, we revisit this problem under the condition of ergodic stationary cross-traffic arrival. We derive the results for both a single-hop path and a multihop path with one-hop persistent cross-traffic routing.

\section{A. Single-Hop Response Curves}

To derive the single-hop results, we assume that the tight link $L_{b}$ is the only link that changes the input packet-pair dispersions. This means that the input dispersion $\delta_{b}$ at the tight link is the same as the input dispersion $\delta$ (or $\delta_{1}$ ) formed by the probing source and that the output dispersion $\delta_{b+1}$ is the same as the output dispersion $\delta^{\prime}$ (or $\delta_{N+1}$ ) at the end of the path, as so obtained by the probing receiver. Then, we have the following closed-form expression for $E\left[\delta^{\prime}\right]$.

Theorem 4: Let $P_{\delta}(x)$ be the distribution function of the random variable $B_{b, \delta}$. When link $b$ is probed by a packet-pair with input dispersion $\delta$ and packet size $s$, the statistical mean of the output dispersion $\delta^{\prime}$ is given by

$$
\begin{aligned}
E\left[\delta^{\prime}\right] & =\frac{\delta \lambda_{b}+s}{C_{b}}+\int_{s / \delta}^{C_{b}} \frac{x \delta-s}{C_{b}} d P_{\delta}(x) \\
& =\delta+\int_{0}^{s / \delta} \frac{s-x \delta}{C_{b}} d P_{\delta}(x) .
\end{aligned}
$$

${ }^{6}$ Recall that $P_{\infty}$ is the step function given in (26).

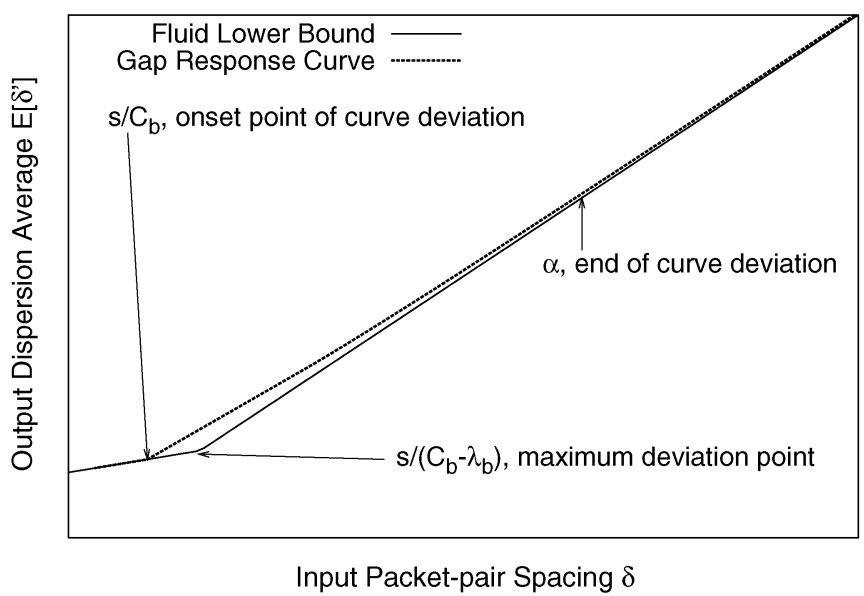

Fig. 5. Illustration of the gap response curve.

Proof: This result directly follows from Theorem 2, Lemma 2, (27), and (28).

Note that (38) is different from the fluid model (3). In fact, as schematically showed in Fig. 5, the fluid model is a lower bound of the real curve. In the input dispersion range $\left(s / C_{b}, \alpha\right)$, the real curve positively deviates from this lower bound and reaches the maximum deviation at the point $\delta=s /\left(C_{b}-\lambda\right)$, where the input rate is equal to the available bandwidth. This response deviation is also illustrated by the curve in the shadow area of Fig. 4 and can be expressed as

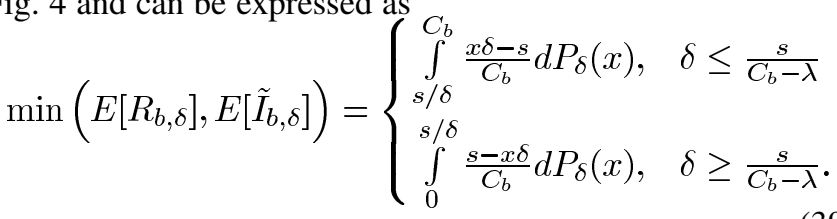

This result tells us about the deciding factors of the response deviation. A precise computation is, however, difficult due to the unknown available bandwidth distribution function $P_{\delta}(x)$ in practice.

It helps to identify the exact value of $\alpha$, which represents the end point of the deviation range. Note that $\alpha$ is the minimum input dispersion that makes $R_{b, \delta}=0$ with probability one

$$
\begin{aligned}
\alpha & =\inf \left\{\delta: E\left[R_{b, \delta}\right]=0\right\} \\
& =\inf \left\{\delta: \operatorname{Prob}\left(B_{b, \delta}<\frac{s}{\delta}\right)=0\right\} \\
& =\inf \left\{\delta: P_{\delta}\left(\frac{s}{\delta}\right)=0\right\} .
\end{aligned}
$$

This requires that $B_{b, \delta}$ be greater than the input probing rate $s / \delta$ almost surely. In other words, the input rate $s / \delta$ must be smaller than the distribution lower bound of $\delta$-interval available bandwidth at link $b$. It is often not possible to satisfy such a condition exactly, ${ }^{7}$ since the convergence of $P_{\delta}(x)$ to the step function in (26) might only be asymptotic and $P_{\delta}(x)$ may remain positive for all $x \in(0, C]$ regardless of the observation interval $\delta$. In that case, $\alpha=\infty$ and we can only mark it approximately at a point where the deviation becomes practically negligible. It is often more informative to look at the rate version of the response curve rather than the spacing version, because the rate response curve has a direct association with cross-traffic arrival rate and 


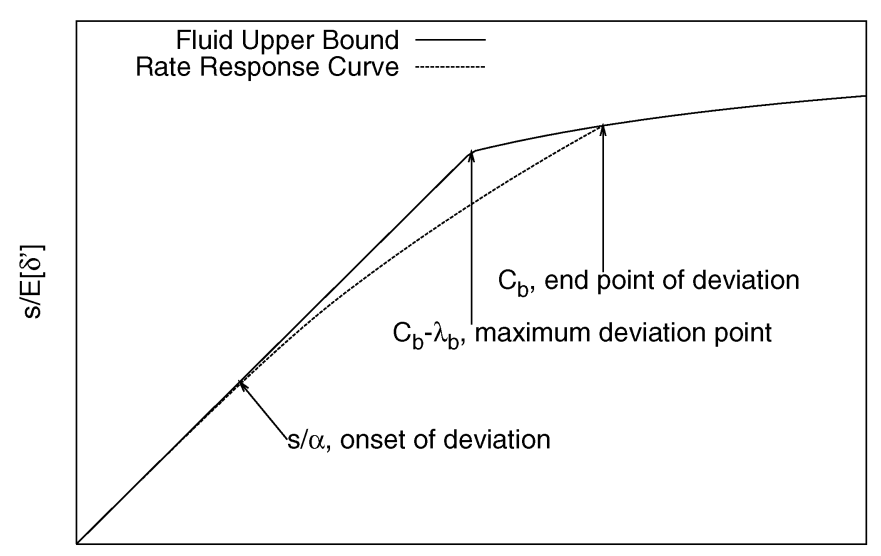

Input Rate $r$

Fig. 6. Illustration of the rate response curve.

available bandwidth. Plotting $s / E\left[\delta^{\prime}\right]$ with respect to $r=s / \delta$ and comparing it with the fluid model (2), we get Fig. 6. The fluid rate model becomes an upper bound of the real response curve. The input rate range $\left(s / \alpha, C_{b}\right)$ becomes the area where the real curve negatively deviates from the fluid model.

\section{B. Multihop Probing Response Curve}

We next take into account the effects of nontight links and derive the packet-pair response curve for an $N$-hop path. The input packet-pair dispersion is a deterministic constant $\delta_{1}$, the output dispersion $\delta_{i+1}$ (at link $i$ ) now becomes random, for all $1 \leq i \leq N$. The multihop gap response curve of the path is the functional relation between $E\left[\delta_{N+1}\right]$ and $\delta_{1}$, which has the following recursive expression:

$$
\begin{aligned}
E\left[\delta_{i+1}\right] & =\frac{E\left[\delta_{i}\right] \lambda_{i}+s}{C_{i}}+E\left[\tilde{I}_{i, \delta_{i}}\right] \\
& =E\left[\delta_{i}\right]+E\left[R_{i, \delta_{i}}\right] .
\end{aligned}
$$

The exact expressions for $E\left[\tilde{I}_{i, \delta_{i}}\right]$ and $E\left[R_{i, \delta_{i}}\right]$ would depend on the distribution of $\delta_{i}$, as we mentioned in the previous section. Suppose that the distribution function of $\delta_{i}$ is $P_{i}(\delta)$. Then, we can compute the two terms by conditioning on $\delta_{i}=\delta$

$$
\begin{aligned}
E\left[\tilde{I}_{i, \delta_{i}}\right] & =\int_{0}^{\infty} E\left[\tilde{I}_{i, \delta}\right] d P_{i}(\delta) \\
& =\int_{0}^{\infty} \int_{s / \delta}^{C_{i}} \frac{x \delta-s}{C_{i}} d P_{\delta}(x) d P_{i}(\delta) \\
E\left[R_{i, \delta_{i}}\right] & =\int_{0}^{\infty} E\left[R_{i, \delta}\right] d P_{i}(\delta) \\
& =\int_{0}^{\infty} \int_{0}^{s / \delta} \frac{s-x \delta}{C_{i}} d P_{\delta}(x) d P_{i}(\delta) .
\end{aligned}
$$

To compare the multihop curve (41) with its fluid counterpart, recall that the multihop response curve in constant-rate fluid cross-traffic can be recursively expressed in the following,

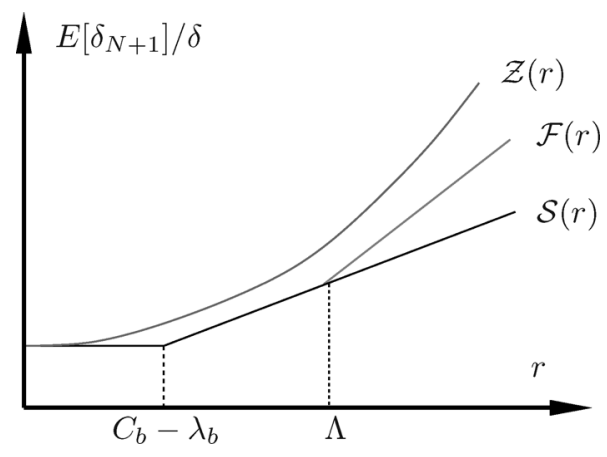

Fig. 7. Illustration of the transformed versions of the real packet-pair rate response curve $\mathcal{Z}(r)$, the multihop fluid rate curve $\mathcal{F}(r)$, and the single-hop fluid rate curve $\mathcal{S}(r)$.

where we use $g_{i}$ to represent the input dispersion at link $i$ to differentiate from $\delta_{i}$ used in bursty cross-traffic

$$
g_{i+1}=\max \left(g_{i}, \frac{\lambda_{i} g_{i}+s}{C_{i}}\right)= \begin{cases}\frac{\lambda_{i} g_{i}+s}{C_{i}}, & g_{i} \leq \frac{s}{C_{i}-\lambda_{i}} \\ g_{i}, & g_{i} \geq \frac{C_{i}-\lambda_{i}}{4}\end{cases}
$$

Denoting by $\beta_{i}$ the difference $E\left[\delta_{i}\right]-g_{i}$, we can obtain the following recursive expression of $\beta_{i}$, based on (41) and (44), where $\beta_{1}=\delta_{1}-g_{1}=0$ :

$$
\beta_{i+1}=\left\{\begin{array}{ll}
\beta_{i}+E\left[R_{i, \delta_{i}}\right], & g_{i} \geq \frac{s}{C_{i}-\lambda_{i}} \\
\frac{\beta_{i} \lambda_{i}}{C_{i}}+E\left[\tilde{I}_{i, \delta_{i}}\right], & g_{i} \leq \frac{s}{C_{i}-\lambda_{i}}
\end{array} .\right.
$$

To better understand this result, we expand (45) to a nonrecursive form in two special cases. In the first case, the input rate is less than the path available bandwidth $C_{b}-\lambda_{b}$. That is, we let $\delta_{1}=g_{1}>s /\left(C_{b}-\lambda_{b}\right)$. In such a case, the dispersion $g_{i}$ is always no less than $s /\left(C_{i}-\lambda_{i}\right)$ and $\beta_{N+1}$ can be expressed as

$$
\beta_{N+1}=\sum_{i=1}^{N} E\left[R_{i, \delta_{i}}\right]
$$

In the second case, we consider the input rate larger than the tight link available bandwidth but smaller than any other hop available bandwidth. Consequently, the condition $g_{i}<s /\left(C_{i}-\right.$ $\left.\lambda_{i}\right)$ is true only at the tight link when $i=b$. The deviation $\beta_{N+1}$ in this situation can be expressed as

$$
\beta_{N+1}=\frac{\lambda_{b}}{C_{b}} \sum_{i=1}^{b-1} E\left[R_{i, \delta_{i}}\right]+E\left[\tilde{I}_{b, \delta_{b}}\right]+\sum_{i=b+1}^{N} E\left[R_{i, \delta_{i}}\right] .
$$

To illustrate the multihop curves, we adopt a transformed rate version that exhibits piecewise linearity in fluid cross-traffic models. We define $\mathcal{F}(r)$ to be the function between the ratio $g_{N+1} / g_{1}$ (which is also the ratio between the input and output rate) and the input rate $r=s / g_{1}$ in the fluid model. The transformed version of the single-hop fluid rate curve associated with the tight link is denoted by $\mathcal{S}(r)$. For the real response curve, we use $\mathcal{Z}(r)$ to denote the relation between $E\left[\delta_{N+1}\right] / \delta_{1}$ and the input rate $r=s / \delta_{1}$. Fig. 7 illustrate these three response curves, Where we see that the real curve $\mathcal{Z}(r)$ appears above $\mathcal{F}(r)$ in the entire input rate range. The amount of deviation is $\beta_{N+1} / g_{1}$. 
Also, note that $\mathcal{F}(r)$ is a piecewise linear curve with the first two linear segments overlapping with $\mathcal{S}(r)$, and the other linear segments appearing above $\mathcal{S}(r)$.

\section{Impact of Probing Packet Size}

How does the packet size $s$ affect the response curves or the amount of response deviation from fluid models? To answer this question, we examine the rate response curve evolving tend when the probing packet size $s$ increases. For any given input probing rate $r$, we denote by $\delta_{i+1}(r, s)$ the packet-pair output dispersion random variable at link $i$, and denote by $g_{i+1}(r, s)$ the deterministic output dispersion at link $i$ when cross-traffic flows are constant-rate fluid. Then, the following theorem states a condition under which the response deviation vanishes when $s \rightarrow \infty$.

Theorem 5: Assuming that for each link $1 \leq i \leq N$, the distribution function of $B_{i, \delta}$, denoted by $P_{i, \delta}(r)$, converges to the step function $P_{\infty}(r)$ in (26) with the following speed:

$$
P_{i, \delta}(r)-P_{\infty}(r)=o\left(\frac{1}{\delta^{2}}\right)
$$

then the response deviation decays to 0 as $s$ increases

$$
\lim _{s \rightarrow \infty} E\left[\delta_{N+1}(r, s)-g_{N+1}(r, s)\right]=0 .
$$

The asymptotic variance of $\delta_{N+1}$ when $s$ increases is upper bounded by some constant $K_{N+1}$

$$
\lim _{s \rightarrow \infty} E\left[\left(\delta_{N+1}(r, s)-g_{N+1}(r, s)\right)^{2}\right] \leq K_{N+1} .
$$

Proof: Please refer to Appendix I.

Note that for a single-hop path, condition (48) can be relaxed to a convergence speed of $o(1 / \delta)$. Even though these conditions appear cryptic, they are valid in a broad range of cross-traffic environments. In particular, when cross-traffic arrivals result in a regenerative queue (which is very common both in stochastic modeling and in practice), Theorem 6 shows that the convergence speed of $P_{i, \delta}(r)$ to $P_{\infty}(r)$ is in fact exponential, much faster than required in Theorem 5. Hence, large probing packet size usually implies less deviation of the real response curve from its fluid counterpart.

Theorem 6: When hop utilization process $\left\{U_{i}(t)\right\}$ is regenerative, ${ }^{8} P_{i, \delta}(r)-P_{\infty}(r)$ is an asymptotically exponential function of $\delta$. That is, there exists a positive constant $k$, such that

$$
P_{i, \delta}(r)-P_{\infty}(r)=\Theta\left(e^{-k \delta}\right) .
$$

Proof: Please refer to [13].

It is beneficial to have some extra understanding of Theorem 5 using the transformed rate response curves plotted in Fig. 7. Note that as $s \rightarrow \infty$, the curve $\mathcal{Z}$ approaches $\mathcal{F}$ in the entire input rate range, which also means that the single-hop curve $\mathcal{S}$ is a tight lower bound of $\mathcal{Z}$ in the input rate range $(0, \Lambda)$, but not in the rate range $(\Lambda, \infty)$.

In practice, however, due to the limit of path MTU and the concern of packet fragmentation, probing packet size $s$ can not be made arbitrarily large. The question becomes whether the commonly used MTU of 1500 bytes is enough to reduce

${ }^{8}$ Refer to [24, p. 89] for the definition of regenerative processes.

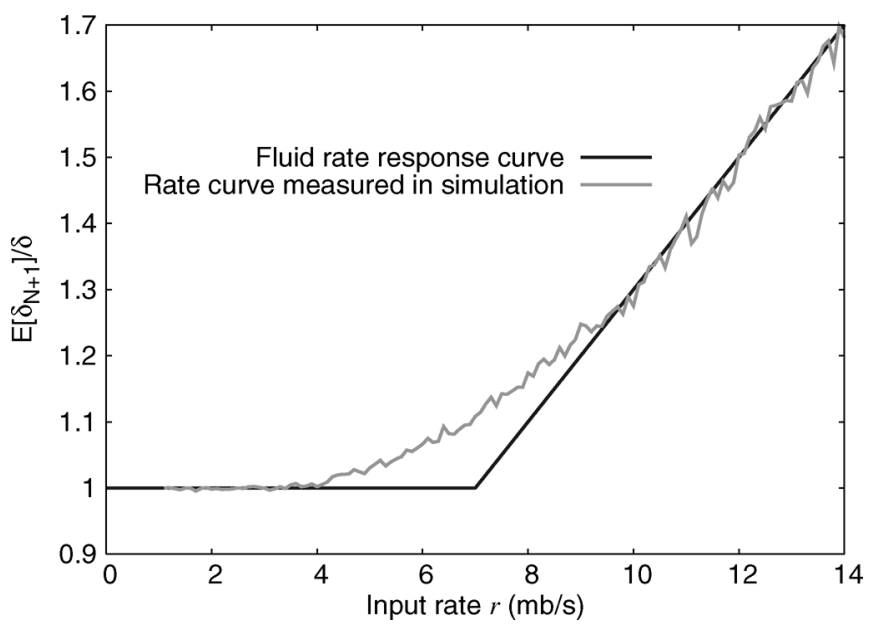

Fig. 8. Transformed versions of the fluid rate curve and the real rate curve measured in simulation.

the amount of curve deviation to such an extent that its impact on bandwidth measurement accuracy becomes insignificant? In Section VI, we will use several experiments to show that the response deviation phenomenon have a significant adverse impact on bandwidth estimation even when the probing packet size $s$ is set to be the largest possible.

\section{Packet-Train Probing}

We have been focusing on the case of packet-pair sampling in this paper. Our analytical approach can be extended to understand packet-train probing techniques such as pathload and IGI/PTR. This extension, even though logically straightforward, comes at the cost of substantially more complex presentations. Due to limited space, we next present the main result without elaboration, and refer interested readers to [14] for more details.

Recall that a packet-train usually consists of $n$ packets (packet-pair is a special case when $n=2$ ) and that the dispersion of a packet-train is defined as the average interpacket time spacing within the train. If we view the first and the last packets in the train as a packet-pair and denote by $\delta_{i+1}$ the output dispersion of this pair at link $i$, then, the output dispersion $G_{i+1}$ of the train at link $i$ is equal to $\delta_{i+1} /(n-1)$. Under certain additional assumptions, we showed in [14] that as packet-train length $n$ increases, the output dispersion $G_{N+1}$ converges to its fluid counterpart $g_{N+1}$ in the mean-square sense, for any input dispersion (at the first link) and any probing packet size

$$
\lim _{n \rightarrow \infty} E\left[\left(G_{N+1}-g_{N+1}\right)^{2}\right]=0 .
$$

This result shows that using a packet-train can mitigate the adverse impact of the response deviation phenomenon on bandwidth estimation. It explains the reason why packet-train bandwidth estimation tools (e.g., pathload and PTR) are usually much more accurate than packet-pair tools.

\section{IMPACT OF RESPONSE CURVE DEVIATION ON BANDWIDTH MEASUREMENT}

In this section, we first quantitatively observe the packet-pair response deviation in both single-hop and multihop paths. We 


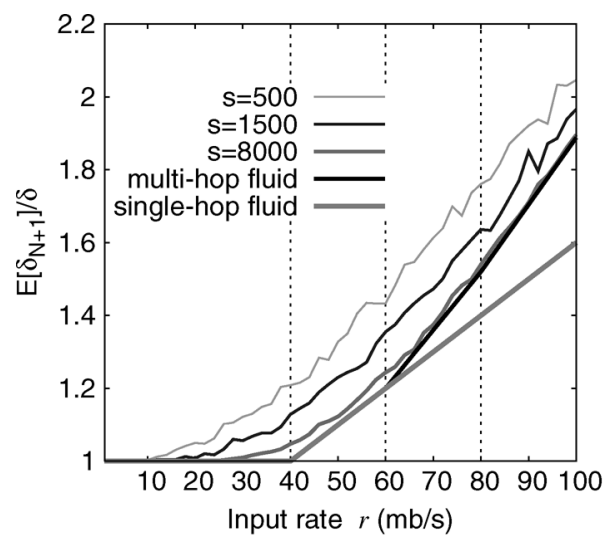

(a)

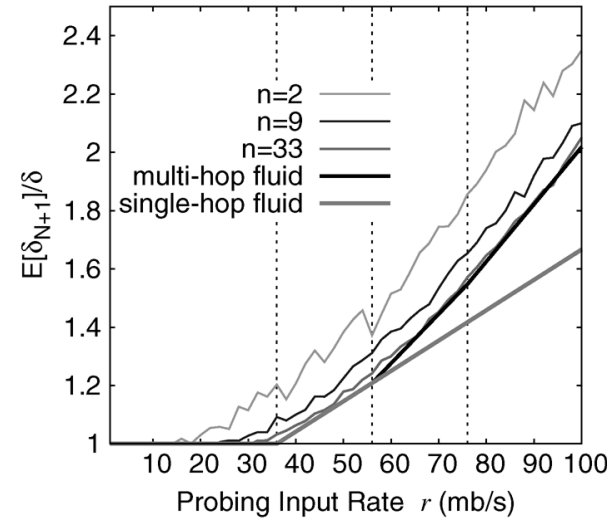

(b)

Fig. 9. Multihop response curves. (a) Using packet-pairs of different size. (b) Using packet-trains of different length.

then show how this phenomenon adversely affects the accuracy of two representative measurement techniques, TOPP and Spruce.

\section{A. Single-Hop Experiments}

In our first experiment, we use a single-hop path with capacity $C=10 \mathrm{mb} / \mathrm{s}$. We use Poisson cross-traffic with average arrival rate $\lambda=3 \mathrm{mb} / \mathrm{s}$. The probing packet size is chosen to be 1500 bytes. We compute $E\left[\delta^{\prime}\right]$ for 130 input values of $\delta$ in [0.86 and $12 \mathrm{~ms}$ ], which corresponds to 130 equally spaced input rates in the range of [ 1.0 and $14 \mathrm{mb} / \mathrm{s}]$. We obtain the response curves using an NS2 simulation experiment. In the simulation, the sender transmits 1000 packet-pairs for each input rate. The interprobing delay is controlled by a random variable with its mean set to ten times as large as the input packet-pair dispersion $\delta$. We use the average of the 1000 output dispersions to approximate $E\left[\delta^{\prime}\right]$.

Fig. 8 shows the transformed versions of both the rate curve measured in NS2 simulation and the fluid rate curve. We can see that the measured curve non-negligibly deviates from the fluid lower bound within the input rate range roughly from 4 to $10 \mathrm{mb} / \mathrm{s}$. The deviation is very clear even though we are using the largest probing packet size. Also, recall that the response curve has provable monotonicity and continuity. Hence, any roughness as we see in the obtained curve comes from measurement errors.

\section{B. Multihop Experiments}

We first use a simple three-hop path in NS2 simulation. The capacity of all the links are $100 \mathrm{mb} / \mathrm{s}$. The cross-traffic flows traversing each link are generated using three NLANR [1] traces with average arrival rates $\lambda_{1}=20, \lambda_{2}=40$, and $\lambda_{3}=60 \mathrm{mb} / \mathrm{s}$. All interpacket delays in each trace are scaled by a common factor so that the average rate during the trace duration becomes the desired value. The trace durations after scaling are 1-2 minutes, and the trace is replayed once the previous round is finished. In these settings, link $L_{3}$ is the tight link of the path with available bandwidth $40 \mathrm{mb} / \mathrm{s}$.

To obtain the packet-pair response curves while at the same time examine the impact of probing packet size and confirm the result in Theorem 5, we use packet-pairs of different sizes to measure the rate response curves. For each packet size, we probe the path at 45 input rates, from 10 to $100 \mathrm{mb} / \mathrm{s}$ with $2 \mathrm{mb} / \mathrm{s}$ increasing step. For each input rate, we use 500 packet-pairs to estimate $E\left[\delta_{4}\right]$, the mean of the output dispersions.

Fig. 9(a) plots the transformed rate curves for probing packet size 500, 1500, and 8000 (all in bytes). ${ }^{9}$ To compare with the fluid models. We also plot both the multihop fluid curve (computed recursively using the single-hop model) and the single-hop fluid curve at the tight link (i.e, $L_{3}$ ). Note that the multihop fluid curve is composed of four linear segments with turning points located at 40,60, and $80 \mathrm{mb} / \mathrm{s}$. The single-hop fluid curve is composed of two linear segments with a turning point at $40 \mathrm{mb} / \mathrm{s}$, which is the path available bandwidth. The two fluid curves are independent of the probing packet size and they coincide with each other in the input rate range $(0$, 60). In the input rate range from 60 to $100 \mathrm{mb} / \mathrm{s}$, The multihop fluid curve appears above the single-hop fluid curve. The three real rate curves measured in NS2 simulations all appear above the multihop fluid curve, which they approach as the probing packet size increases. The curve measured using a packet size of 1500 bytes shows a significant deviation from its fluid counterpart. For $s=8000$ bytes, the measured curve almost coincides with the multihop fluid curve in the input rate range $(60,100)$. However, in the input range $(30,60)$, the amount of the deviation is still clearly noticeable.

To understand packet-train probing response curves and verify the results in Section V-D, we probe using packet-trains a three-hop Emulab network path that has the same settings and traversing cross-traffic flows. All parameters used in the testbed experiment (except for the train length) are the same as those used in the previous ns 2 simulation. Fig. 9(b) shows the measured curves for $n=2,9$, and 33. We observe that as packet-train length increases, the measured response curve converges from above to the multihop fluid lower bound. Noticed that in the Emulab path, the IP-level capacity of each Ethernet path is $96 \mathrm{mb} / \mathrm{s}$. Hence, the turning points on the fluid curves all have a left shift of $4 \mathrm{mb} / \mathrm{s}$, compared with those in the ns2 simulation.

${ }^{9}$ Note that such an experiment can not be replicated in real networking testbed because a packet size as large as 8000 bytes will cause fragmentation, which is avoided in NS2 simulations. 
TABLE II

TOPP ESTIMATION RESULTS (IN mb/s)

\begin{tabular}{llll}
\hline & $C_{b}$ & $\lambda_{b}$ & $C_{b}-\lambda_{b}$ \\
\hline Real values & 10 & 3.0 & 7.0 \\
TOPP in ns2 simulation & 35.97 & 32.33 & 3.64 \\
\hline
\end{tabular}

Next, we discuss the implications of our findings on two packet-pair bandwidth measurement techniques: TOPP and Spruce.

\section{TOPP}

TOPP measures the tight link bandwidth characteristics from the transformed multihop fluid rate curve, assuming that it is a valid first-order approximation of the real response curve. TOPP identifies the second linear segment from the transformed rate curve and applies linear regression to calculate the capacity $C_{b}$ and cross-traffic intensity $\lambda_{b}$.

We first consider the single-hop response curves in Fig. 8. We see that the second segment falls into the deviated range. When applying linear regression on this segment, we get estimation results in Table II. The table shows that even if TOPP could manage to obtain a precise rate curve, it would not achieve an accurate measurement due to its unawareness of the deviation phenomenon.

In a single-hop case, note that the real curve agrees with fluid model when $r \geq C_{b}$. Therefore, linear regression can be applied to this curve portion to extract the capacity $C_{b}$ and cross-traffic intensity $\lambda_{b}$. In a multihop path, the response curve usually deviates from its fluid counterpart in the entire range and does not necessarily exhibit piece-wise linearity, as exemplified by Fig. 9. Hence, any attempt to search for the second linear segment from the curve can lead to measurement errors even more significant than what we showed in Table II.

\section{Spruce}

Spruce measures the path available bandwidth based on the single-hop fluid response curve at the input rate $r=C_{b}$. Spruce assumes that the tight link capacity is known beforehand and that nontight links have negligible effects on the measurement accuracy. Using the response curve notations we introduced in Section V, the Spruce available bandwidth estimator can be written as

$$
C_{b}\left(1-\frac{E\left[\delta^{\prime}\right]-\delta}{\delta}\right)=C_{b}\left(2-\mathcal{Z}\left(C_{b}\right)\right)
$$

Our first observation is that Spruce estimator is unbiased if and only if the following condition holds:

$$
\mathcal{Z}\left(C_{b}\right)=\mathcal{S}\left(C_{b}\right)
$$

where $\mathcal{S}\left(C_{b}\right)=1+\lambda_{b} / C_{b}$ is the transformed single-hop fluid rate response at input rate $C_{b}$. This condition holds in a single-hop path regardless of the probing packet size, as we showed in Section V. Hence, Spruce estimator is unbiased in a single-hop path. In a multihop path, however, $\mathcal{Z}\left(C_{b}\right)$ is usually
TABLE III

SPRUCE BIAS IN MULTIHOP EMULAB EXPERIMENT (IN mb/s)

\begin{tabular}{|cccc|}
\hline train length & elastic bias & non-elastic bias & total bias \\
\hline 2 & 54 & 30 & 84 \\
\hline 9 & 10 & 30 & 40 \\
\hline 33 & 0 & 30 & 30 \\
\hline
\end{tabular}

larger than $\mathcal{S}\left(C_{b}\right)$, as illustrated in Fig. 9. This causes a negative bias (underestimation) in Spruce's available bandwidth estimation. The amount of bias is given by

$$
C_{b}\left(\mathcal{Z}\left(C_{b}\right)-\mathcal{F}\left(C_{b}\right)\right)+C_{b}\left(\mathcal{F}\left(C_{b}\right)-\mathcal{S}\left(C_{b}\right)\right) .
$$

The first additive term in (55) is the measurement bias caused by the curve deviation of $\mathcal{Z}$ from $\mathcal{F}$ at input rate $C_{b}$, which, as stated in Section $\mathrm{V}$, will vanish when the probing packet size $s \rightarrow \infty$ or when long packet-trains are used. Hence, we call it elastic bias. The second additive term is the portion of measurement bias caused by the curve deviation of $\mathcal{F}$ from $\mathcal{S}$ at input rate $C_{b}$, which remains constant for any packet size $s$ or any long packet-trains. Therefore, it is nonelastic bias. Conceptually, elastic bias stems from cross-traffic burstiness and nonelastic bias is a consequence of multihop effects.

In Table III, we give the amount of measurement bias caused by the two types of curve deviations in our multihop Emulab packet-train probing experiment. Using a train length of 2 (i.e., packet-pair), the elastic bias is $54 \mathrm{mb} / \mathrm{s}$, about $150 \%$ of the actual available bandwidth. We also see that increasing the train length to 33 can completely overcome the elastic bias, but can not change the nonelastic bias of $30 \mathrm{mb} / \mathrm{s}$.

\section{CONCLUDING REMARKS}

In this paper, we presented a "sampling-and-constructing" view to understand the essence of packet-pair probing. We identified several types of important information about the queueing system that are sampled and encoded in packet-pair dispersions. Our approach uncovers the full picture of packet-pair sampling and leads to a closed-form solution to the multihop response curve, which extends previous fluid models and serves as a theoretical foundation for active bandwidth measurement.

To convey the main idea and the spirit of our analytical approach, we limited the scope of our discussions in several aspects. Note that the major results in this paper can be generalized along several directions. In particular, we extended our discussion to packet-train sampling and relaxed the cross-traffic stationarity assumption in [12]. We also accommodated arbitrary cross-traffic routing into the multihop analysis in [14]. We refer interested readers to [12] and [14] for additional results, but point out that they also use a similar analytical approach that features the "sampling-and-constructing" characterization of active packet probing. In our future work, we are interested in developing new techniques that overcome the problems we have identified in existing tools.

\section{APPENDIX I}

\section{PROOF OF THEOREM 5}

We first present the following lemma. 
Lemma 3: Given (48), when link $L_{i}$ is probed by a packetpair with an input rate $r$, we have the following two limits regarding the second-order moments of $R_{i, s / r}$ and $\tilde{I}_{i, s / r}$ :

$$
\left\{\begin{array}{ll}
\lim _{s \rightarrow \infty} E\left[R_{i, s / r}^{2}\right]=0, & r<C_{i}-\lambda_{i} \\
\lim _{s \rightarrow \infty} E\left[\tilde{I}_{i, s / r}^{2}\right]=0, & r>C_{i}-\lambda_{i}
\end{array} .\right.
$$

Proof: We only consider the case when $r<C_{i}-\lambda_{i}$ and prove the first part of (56). The proof for the second part is similar. Note that

$$
R_{i, \delta}=\max \left(0, \frac{s-\delta B_{i, \delta}}{C_{i}}\right) .
$$

Denoting by $P_{i, \delta}(x)$ the distribution function of $B_{i, \delta}$, we get the second-order moment of (57) as

$$
E\left[R_{i, \delta}^{2}\right]=\int_{0}^{r} \frac{\delta^{2}(r-x)^{2}}{C_{i}^{2}} d P_{i, \delta}(x) \leq \frac{2 r^{2} \delta^{2} P_{i, \delta}(r)}{C_{i}^{2}} .
$$

Taking the limit of (58) and further recalling (48), we get

$$
0 \leq \lim _{\delta \rightarrow \infty} E\left[R_{i}^{2}(\delta)\right] \leq \lim _{\delta \rightarrow \infty} \frac{2 r^{2} \delta^{2} P_{i, \delta}(r)}{C_{i}^{2}}=0 .
$$

This leads to the first part in (56).

We next prove Theorem 5.

Proof: We apply mathematical induction to $i$. For the base case when $i=1, \delta_{1}=s / r=g_{1}$ and $K_{1}=0$, the theorem holds trivially. Suppose that the theorem holds for $i=N$, then we next show that it also holds for $i=N+1$.

First, consider the case when $s / g_{N}<C_{N}-\lambda_{N}$. Recalling Theorem 2, we have

$$
\delta_{N+1}=\delta_{N}+D_{N, \delta_{N}}+R_{N, \delta_{N}} .
$$

We now examine the asymptotic mean and asymptotic variance of each term on the right-hand side of (60). For the first term $\delta_{N}$, due to the induction hypothesis, we have

$$
\begin{aligned}
\lim _{s \rightarrow \infty} E\left[\delta_{N}\right] & =g_{N} \\
\lim _{s \rightarrow \infty} E\left[\left(\delta_{N}-g_{N}\right)^{2}\right] & \leq K_{N} .
\end{aligned}
$$

The second term in (60) is a zero-mean random variable. That is

$$
\lim _{s \rightarrow \infty} E\left[D_{N, \delta_{N}}\right]=0 .
$$

The variance of $D_{N, \delta_{N}}$ converges to $2 \operatorname{Var}\left[W_{N}\right]$ as $s \rightarrow \infty$, which is a constant with respect to $s$. To show this, first note that

$$
E\left[\left(D_{N, \delta_{N}}\right)^{2}\right]=\int_{0}^{\infty}\left(D_{N, x}\right)^{2} d P(x)
$$

where $P(x)$ is the distribution function of $\delta_{N}$. The integral term in (64) can be decomposed into the sum of three integral terms as follows:

$$
E\left[D^{2}\right]=\int_{0}^{E / 2} D^{2} d P(x)+\int_{E / 2}^{3 E / 2} D^{2} d P(x)+\int_{3 E / 2}^{\infty} D^{2} d P(x)
$$

where $D^{2}=\left(D_{N, x}\right)^{2}$ and $E=E\left[\delta_{N}\right]$. Using Chebyshev's inequality and the fact that $E\left[D^{2}\right] \leq 4 \operatorname{Var}\left[W_{N}\right]$, it is easy to show that both the first and the third integral terms in (65) converges to 0 as $s \rightarrow \infty$. In addition, using Chebyshev's inequality, we can show that the second integral term in (65) converges to $2 \operatorname{Var}\left[W_{N}\right]$ as $s \rightarrow \infty$. Omitting the intermediate steps, we get

$$
\lim _{s \rightarrow \infty} E\left[\left(D_{N, \delta_{N}}\right)^{2}\right]=2 \operatorname{Var}\left[W_{N}\right] .
$$

For the third term $R_{N, \delta_{N}}$ in (60), its first-order moment converges to 0 as $s \rightarrow \infty$ as we show next. Note that

$$
E\left[R_{N, \delta}\right]=\int_{0}^{s / A_{N}} E\left[R_{N, x}\right] d P(x)+\int_{s / A_{N}}^{\infty} E\left[R_{N, x}\right] d P(x)
$$

where $A_{N}=C_{N}-\lambda_{N}$ is the available bandwidth of $L_{N}, P(x)$ is the distribution function of $\delta_{N}$. Notice that $R_{N, \delta_{N}}$ is upper bounded by $s / C_{N}$. Hence, due to Chebyshev's inequality, for the first additive term in (67), we have

$$
\begin{aligned}
0 & \leq \int_{0}^{s / A_{N}} E\left[R_{N, x}\right] d P(x) \leq \frac{s}{C_{N}} P\left(\frac{s}{A_{N}}\right) \\
& \leq \frac{s \operatorname{Var}\left[\delta_{N}\right]}{C_{N}\left(s / A_{N}-E\left[\delta_{N}\right]\right)^{2}} .
\end{aligned}
$$

Taking the limit of (68) when $s \rightarrow \infty$, we get

$$
\begin{aligned}
0 & \leq \lim _{s \rightarrow \infty} \int_{0}^{s / A_{N}} E\left[R_{N, x}\right] d P(x) \\
& \leq \lim _{s \rightarrow \infty} \frac{s}{C_{N}} \frac{\operatorname{Var}\left[\delta_{N}\right]}{\left(s / A_{N}-E\left[\delta_{N}\right]\right)^{2}} \\
& \leq \lim _{s \rightarrow \infty} \frac{s}{C_{N}} \frac{K_{N}}{\left(s / A_{N}-g_{N}\right)^{2}}=\lim _{s \rightarrow \infty} \Theta\left(\frac{1}{s}\right)=0
\end{aligned}
$$

where the last inequality is due to the induction hypothesis and the second last equality is due to the fact that $g_{N}$ is a linear function of $s / r$.

For the second additive term in (67), first recall Theorem 3, which says that $R_{N, x}$ is a monotonically decreasing function of $x$. Therefore, we have

$$
\begin{aligned}
0 & \leq \lim _{s \rightarrow \infty} \int_{s / A_{N}}^{\infty} E\left[R_{N, x}\right] d P(x) \\
& \leq \lim _{s \rightarrow \infty} E\left[R_{N, s / A_{N}}\right]=0
\end{aligned}
$$


where the last equality is due to Lemma 3, which implies that $R_{N}\left(s / A_{N}\right)$ also converges to 0 in mean when $s \rightarrow \infty$. Combining (69) and (70), it follows that:

$$
\lim _{s \rightarrow \infty} E\left[R_{N, \delta_{N}}\right]=0 .
$$

Similar to the transition from (67) to (71), we can prove that the asymptotic variance of $R_{N, \delta_{N}}$ when $s$ increases is bounded by a constant. We omit the proof details of this step. Combining all these investigations, it follows that:

$$
\lim _{s \rightarrow \infty} E\left[\delta_{N+1}\right]=\lim _{s \rightarrow \infty} E\left[\delta_{N}\right]=g_{N}=g_{N+1} .
$$

The asymptotic variance of $\delta_{N+1}$ is also bounded by a constant irrespective of $s$ due to the fact that all the additive terms on the right-hand side of (60) have bounded asymptotic variance. We denote this variance upper bound by $K_{N+1}$.

So far, we finished the proof for the case when $s / g_{N}<A_{N}$. For the case when $s / g_{N}>A_{N}$, we have

$$
\delta_{N+1}=\frac{Y_{N, \delta_{N}} \delta_{N}}{C_{N}}+\frac{s}{C_{N}}+\tilde{I}_{N, \delta_{N}} .
$$

We now examine the asymptotic mean and variance for each of the additive terms on the right-hand side of (73). For the first term, by conditioning on $\delta_{N}$, we get

$$
\lim _{s \rightarrow \infty} E\left[\frac{Y_{N, \delta_{N}} \delta_{N}}{C_{N}}\right]=\frac{\lambda_{N} g_{N}}{C_{N}} .
$$

Similarly, we can also get the asymptotic variance as follows:

$$
\lim _{s \rightarrow \infty} \operatorname{Var}\left[\frac{Y_{N, \delta_{N}} \delta_{N}}{C_{N}}\right]=\frac{\lambda_{N}^{2}}{C_{N}^{2}} \lim _{s \rightarrow \infty} \operatorname{Var}\left[\delta_{N}\right] \leq \frac{\lambda_{N}^{2} K_{N-1}}{C_{N}^{2}}
$$

where the last inequality is due to induction hypothesis. Note that the limiting variance is bounded by a constant that does not depend on $s$.

The second additive term in (73) is a constant. For the third term $\tilde{I}_{N, \delta_{N}}$, we now show that it converges to 0 in the meansquare sense as $s \rightarrow \infty$. Consequently, both the asymptotic mean and the asymptotic variance of this term is 0 . Note that $E\left[\tilde{I}_{N, \delta_{N}}^{2}\right]$ can be decomposed as

$$
\int_{0}^{s / A_{N}} E\left[\tilde{I}_{N, x}^{2}\right] d P_{s}(x)+\int_{s / A_{N}}^{\infty} E\left[\tilde{I}_{N, x}^{2}\right] d P_{s}(x)
$$

where $A_{N}=C_{N}-\lambda_{N}$ is the available bandwidth of $L_{N}, P_{s}(x)$ is the distribution function of $\delta_{N-1}$ given packet size $s$. Note that the first term in (76) approaches 0 as $s \rightarrow \infty$. That is

$$
\begin{aligned}
& \lim _{s \rightarrow \infty} \int_{0}^{s / A_{N}} E\left[\tilde{I}_{N, x}^{2}\right] d P_{s}(x) \\
& =\lim _{s \rightarrow \infty} \int_{A_{N}}^{\infty} E\left[\tilde{I}_{N, s / r}^{2}\right] d \tilde{P}_{s}(r) \\
& \leq \lim _{s \rightarrow \infty} E\left[I_{N, s / A_{N}}^{2}\right]=0
\end{aligned}
$$

where $\tilde{P}_{s}(r)$ is the distribution function of the random variable $s / \delta_{N}$ given that $s$ is fixed. The inequality is due to fact that $\tilde{I}_{N, x}$ is a monotonically decreasing function of $x$ given that $s$ is fixed. The last equality in (77) is due to Lemma 3.

The second term in (76) also approaches 0 as $s \rightarrow \infty$. Note that $\tilde{I}_{N, x} \leq x$, so we have

$$
\begin{gathered}
\lim _{s \rightarrow \infty} \int_{s / A_{N}}^{\infty} E\left[\tilde{I}_{N, x}^{2}\right] d P_{s}(x) \leq \lim _{s \rightarrow \infty} \int_{s / A_{N}}^{\infty} x^{2} d P_{s}(x) \\
=\lim _{s \rightarrow \infty}\left(E\left[\delta_{N}^{2}(s)\right]-\int_{0}^{s / A_{N}} x^{2} d P_{s}(x)\right) \\
=\lim _{s \rightarrow \infty} E\left[\delta_{N}^{2}(s)\right]-\lim _{s \rightarrow \infty} E\left[\delta_{N}^{2}(s)\right]=0 .
\end{gathered}
$$

Combining (77) and (78), it follows that:

$$
\lim _{s \rightarrow \infty} E\left[\tilde{I}_{N, \delta_{N}}^{2}\right]=\lim _{s \rightarrow \infty} E\left[\tilde{I}_{N, \delta_{N}}\right]=0 .
$$

Combining (79) and (74), we have

$$
\lim _{s \rightarrow \infty} E\left[\delta_{N+1}\right]=\frac{\lambda_{N} g_{N}+s}{C_{N}}=g_{N+1} .
$$

Combining induction hypothesis, (75) and (80), we get an upper bound of the asymptotic variance of $\delta_{N+1}$

$$
\lim _{s \rightarrow \infty} \operatorname{Var}\left[\delta_{N+1}\right] \leq \frac{\lambda_{N}^{2}}{C_{N}^{2}} \lim _{s \rightarrow \infty} \operatorname{Var}\left[\delta_{N}\right]=K_{N}
$$

which is a constant independent of $s$. Combining the two cases, we complete the inductive step for any probing input rate $r$. Hence, the theorem follows.

\section{REFERENCES}

[1] National Laboratory for Applied Network Research. [Online]. Available: http://www.nlanr.net

[2] J. Bolot, "Characterizing end-to-end packet delay and loss in the Internet," in Proc. ACM SIGCOMM, 1993, pp. 289-298.

[3] R. Carter and M. Crovella, "Measuring bottleneck link speed in packetswitched networks," Int. J. Perform. Eval., vol. 2728, pp. 273-318, 1996.

[4] C. Dovrolis, P. Ramanathan, and D. Moore, "What do packet dispersion techniques measure?," in Proc. IEEE INFOCOM, Apr. 2001, pp. 905-914.

[5] _ "Packet-dispersion techniques and a capacity estimation methodology," ACM/IEEE Trans. Netw., vol. 12, no. 6, pp. 963-977, Dec. 2004.

[6] K. Harfoush, A. Bestavros, and J. Byers, "Measuring bottleneck bandwidth of targeted path segments," in Proc. INFOCOM, Mar.-Apr. 2003, pp. 2079-2089.

[7] G. He and J. Hou, "On exploiting long range dependence of network traffic in measuring cross traffic on an end-to-end basis," in Proc. IEEE INFOCOM, Mar. 2003, pp. 1858-1868.

[8] N. Hu and P. Steenkiste, "Evaluation and characterization of available bandwidth probing techniques," IEEE J. Sel. Areas Commun., pp. 879-894, Aug. 2003.

[9] V. Jacobson, "Congestion avoidance and control," in Proc. ACM SIGCOMM, 1988, pp. 314-329.

[10] S. Kang, X. Liu, M. Dai, and D. Loguinov, "Packet-pair bandwidth estimation: Stochastic analysis of a single congested node," in Proc. IEEE ICNP, Oct. 2004, pp. 316-325.

[11] K. Lai and M. Baker, "Measuring bandwidth," in Proc. IEEE INFOCOM, 1999, pp. 235-245.

[12] X. Liu, K. Ravindran, B. Liu, and D. Loguinov, "Single-hop probing asymptotics in available bandwidth estimation: Sample-path analysis," ACM IMC, pp. 300-313, Oct. 2004. 
[13] X. Liu, K. Ravindran, and D. Loguinov, "Multi-hop probing asymptotics in available bandwidth estimation: Stochastic analysis," City University of New York (CUNY), New York, Aug. 2005, Tech. Rep..

[14] - "Multi-hop probing asymptotics in available bandwidth estimation: Stochastic analysis," ACM IMC, pp. 186-173, Oct. 2005

[15] _ _ "What signals do packet-pair dispersions carry?," in Proc. IEEE INFOCOM, Mar. 2005, pp. 281-292.

[16] B. Melander, M. Bjorkman, and P. Gunningberg, "A new end-to-end probing and analysis method for estimating bandwidth bottlenecks," in Proc. IEEE GLOBECOM Global Internet Symp., Nov.-Dec. 2000, pp. $415-420$.

[17] _ , "First-come-first-served packet dispersion and implications for TCP," in Proc. IEEE GLOBECOM, 2002, pp. 2170-2174.

[18] _ , "Regression-based available bandwidth measurements," in Proc. SPECTS, Jul. 2002.

[19] A. Pasztor and D. Veitch, "On the scope of end-to-end probing methods," IEEE Commun. Lett., vol. 6, no. 11, pp. 509-511, 2002.

[20] — "The packet size dependence of packet pair like methods," in Proc. IWQoS, 2002, pp. 204-213.

[21] V. Paxson, "End-to-end Internet packet dynamics," IEEE/ACM Trans. Netw., vol. 7, no. 3, pp. 277-292, 1999.

[22] V. Ribeiro, M. Coates, R. Riedi, S. Sarvotham, B. Hendricks, and R. Baraniuk, "Multifractal cross traffic estimation," in ITC Specialist Seminar on IP Traffic Measurement, Sep. 2000.

[23] J. Strauss, D. Katabi, and F. Kaashoek, "A measurement study of available bandwidth estimation tools," ACM IMC, pp. 39-44, 2003.

[24] R. Wolff, Stochastic Modeling and the Theory of Queues. Englewood Cliffs, NJ: Prentice-Hall, 1989.

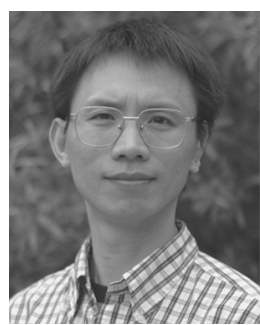

networked systems.

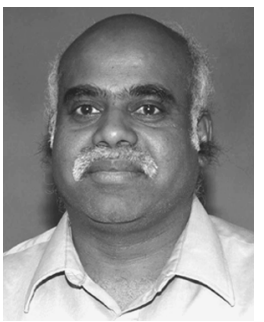

Kaliappa Ravindran received the Ph.D. degree in computer science from the University of British Columbia, Vancouver, BC, Canada.

$\mathrm{He}$ is a faculty member of Department of Computer Science, City University of New York, located in the City College campus. Earlier, he had held faculty positions at Kansas State University, Manhattan, and at the Indian Institute of Science, Bangalore. He had worked in Canadian communication industries for a short period before moving to U.S. His recent project relationships with industries include IBM,

AT\&T, Philips, ITT, and HP. Besides industries, some of his research has been supported by grants and contracts from federal government agencies. His research interests span the areas of service-level management of distributed networks, compositional design of network protocols, system-level support for information assurance, distributed collaborative systems, and Internet architectures.

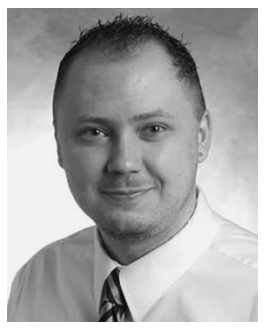

Dmitri Loguinov (S'99-M'03) received the B.S. degree (Hon.) in computer science from Moscow State University, Moscow, Russia, in 1995 and the Ph.D. degree in computer science from the City University of New York, New York, in 2002.

Since September 2002, he has been an Assistant Professor of Computer Science at Texas A\&M University, College Station. His research interests include peer-to-peer networks, Internet video streaming, congestion control, image and video coding, Internet traffic measurement and modeling. Xiliang Liu (S'03-M'05) received the B.S. de-
gree (Hon.) in computer science from Zhejiang University, Hangzhou, China, in 1994, the M.S. degree in information science from the Institute of Automation, Chinese Academy of Sciences, Beijing, China, in 1997, and the Ph.D. degree in computer science from the City University of New York, New York, in 2005.

His research interests include Internet measurement and monitoring, overlay networks, bandwidth estimation, and stochastic modeling and analysis of 